\title{
Network Routing Capacity
}

\author{
Jillian Cannons \\ Elec. \& Comp. Eng. Dept. \\ Univ. of Calif., San Diego \\ La Jolla, CA 92093-0409 \\ Email: jcannons@ucsd.edu
}

\author{
Randall Dougherty \\ Center for Commun. Research \\ 4320 Westerra Court \\ San Diego, CA 92121-1969 \\ Email: rdough@ccrwest.org
}

\author{
Christopher Freiling \\ Department of Mathematics \\ California State University \\ 5500 University Parkway
}

San Bernardino, CA 92407-2397

Email: cfreilin@csusb.edu
Kenneth Zeger

Elec. \& Comp. Eng. Dept.

Univ. of Calif., San Diego

La Jolla, CA 92093-0407

Email: zeger@ucsd.edu

\begin{abstract}
We define the routing capacity of a network to be the supremum of all possible fractional message throughputs achievable by routing. We prove that the routing capacity of every network is achievable and rational, we present an algorithm for its computation, and we prove that every non-negative rational number is the routing capacity of some network. We also determine the routing capacity for various example networks. Finally, we discuss the extension of routing capacity to fractional coding solutions and show that the coding capacity of a network is independent of the alphabet used.
\end{abstract}

\section{INTRODUCTION}

A network is a directed acyclic graph, together with nonempty sets of source nodes, sink nodes, source node messages, and sink node demands. Each message is an arbitrary element of a fixed finite alphabet and is associated with exactly one source node, and each demand at a sink node is a specification of a specific source message that needs to be obtainable at the sink. A network is degenerate if there exists a source message demanded at a particular sink, but no directed path through the graph from the source to the sink.

Each edge in a network carries a vector of symbols from some alphabet. The maximum dimension of these vectors is called the edge capacity. For a given finite alphabet, an edge function is a mapping, associated with a particular edge $(u, v)$, which takes as inputs the edge vector carried on each in-edge to the node $u$ and the source messages generated at node $u$, and produces an output vector to be carried on the edge $(u, v)$. A decoding function is a mapping, associated with a message demanded at a sink, which takes as inputs the edge vector carried on each in-edge to the sink and the source messages generated at the sink, and produces an output vector hopefully equal to the demanded message.

A solution to a network for a given alphabet is an assignment of edge functions to a subset of edges and an assignment of decoding functions to all sinks in the network, such that each sink node obtains all of its demands. A network is solvable if it has a solution for some alphabet. A network solution is a vector routing solution if every edge function is defined so that each component of its output is copied from a (fixed) component of one of its inputs. A network solution is a vector linear solution if every edge function is a linear combination of in-edge vectors, with the coefficients being matrices. A solution is reducible if it has at least one component of an edge function which, when removed, still yields a solution.

A $(k, n)$ fractional routing solution of a network is a vector routing solution that uses messages with $k$ components and edges with capacity $n$. Note that if a network is solvable then it must have a (coding) solution with $k=n=1$. A $(k, n)$ fractional routing solution is minimal if it is not reducible and if no $\left(k, n^{\prime}\right)$ fractional routing solution exists for any $n^{\prime}<n$.

The ratio $k / n$ in a $(k, n)$ fractional routing solution quantifies the capacity of the solution and the rational number $k / n$ is said to be an achievable routing rate of the network. Define the set

$$
U=\{r \in \mathbb{Q}: r \text { is an achievable routing rate }\} .
$$

The routing capacity of a network is the quantity $\epsilon=\sup U$. The routing capacity is said to be achievable if it is an achievable routing rate.

It is known that if a network has a vector routing solution, then it does not necessarily have a scalar routing solution. Similarly, if a network has a vector linear solution, then it does not necessarily have a scalar linear solution [5]. It was noted in [6] that for some networks, the size of the alphabet needed for a solution can be significantly reduced if the solution does not operate at the full capacity of the network. In particular, they demonstrated that, for certain networks, fractional coding can achieve a solution where the ratio of edge capacity $n$ to message vector dimension $k$ is an arbitrarily small amount above one. This paper considers examples and achievability of network routing capacity, and briefly discusses network coding capacity. All proofs and derivations are omitted throughout due to space limitations. ${ }^{1}$

\section{ROUTING CAPACITY OF EXAMPLE NETWORKS}

We first present the routing capacity of some example networks. Let $k$ be the dimension of the messages and let $n$ be the capacity of the edges. Critical edges are labeled to demonstrate a fractional routing solution which achieves the routing capacity.

Example 2.1: (See Figure 1.)

Example 2.2: (See Figure 2.)

Example 2.3: (See Figure 3.) The network $\mathcal{N}_{3}$ contains a

${ }^{1}$ Reference [2] is a full length version of this paper, complete with proofs, and is available on-line at http://code.ucsd.edu/zeger/pubs.html 


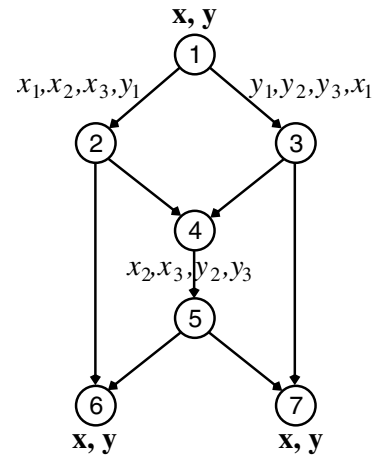

Fig. 1. The multicast network $\mathcal{N}_{1}$ whose routing capacity is $3 / 4$.

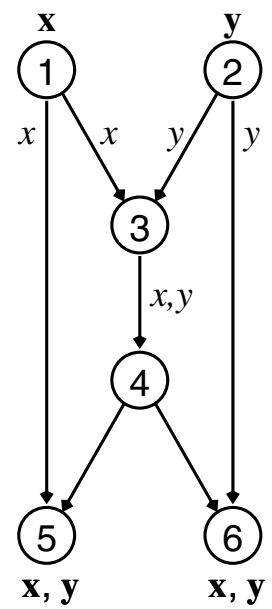

Fig. 2. The network $\mathcal{N}_{2}$ whose routing capacity is $1 / 2$.

single source $n_{1}$ with $m$ messages. The second layer of the network consists of $N$ nodes, each connected to the source via a single edge. The third layer consists of $\left(\begin{array}{l}N \\ I\end{array}\right)$ nodes, each receiving a distinct set of $I$ in-edges from the second layer. Each third layer node demands all messages. The network is linearly solvable if and only if $m \leq I$ (since the network is multicast and the minimum cut size is $I$ for each sink node [4]).

Example 2.4: (See Figure 4.) This network, due to R. Koetter, was used by Médard et al. [5] to demonstrate that there exists a network with no scalar linear solution but with a vector linear solution.

\section{ROUTING CAPACITY ACHIEVABILITY}

We now consider the achievability of network routing capacity. Consider a network and its associated graph $G=(V, E)$, sources $S$, messages $M$, and sinks $K$. For each message $\mathbf{x}$, we say that a directed subgraph of $G$ is an $\mathbf{x}$-tree if the subgraph has exactly one directed path from the source emitting $\mathbf{x}$ to each destination node which demands $\mathbf{x}$, and the subgraph is minimal with respect to this property. For each message $\mathbf{x}$, let $s(\mathbf{x})$ denote the number of $\mathbf{x}$-trees. For a given network and for each message $\mathbf{x}$, let $T_{1}^{\mathbf{x}}, T_{2}^{\mathbf{x}}, \ldots, T_{s(\mathbf{x})}^{\mathbf{x}}$ be an enumeration of all the $\mathbf{x}$-trees in the network.

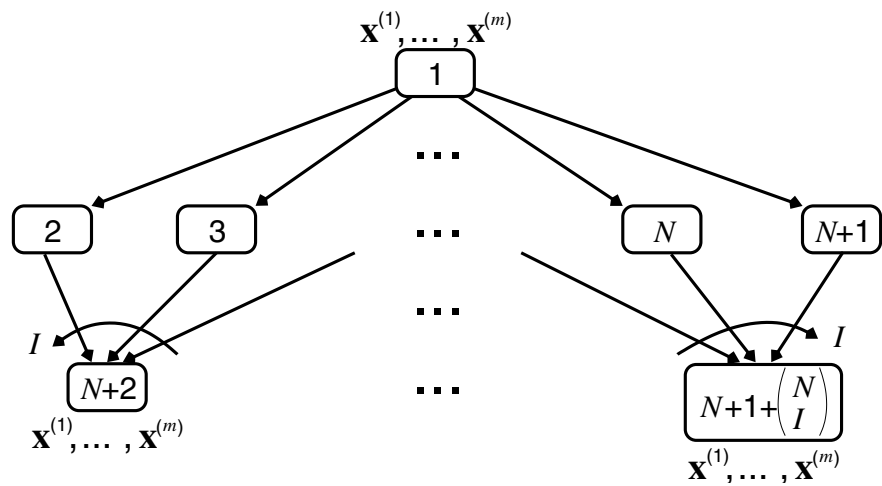

Fig. 3. The multicast network $\mathcal{N}_{3}$ whose routing capacity is $N /(m(N-$ $I+1))$.

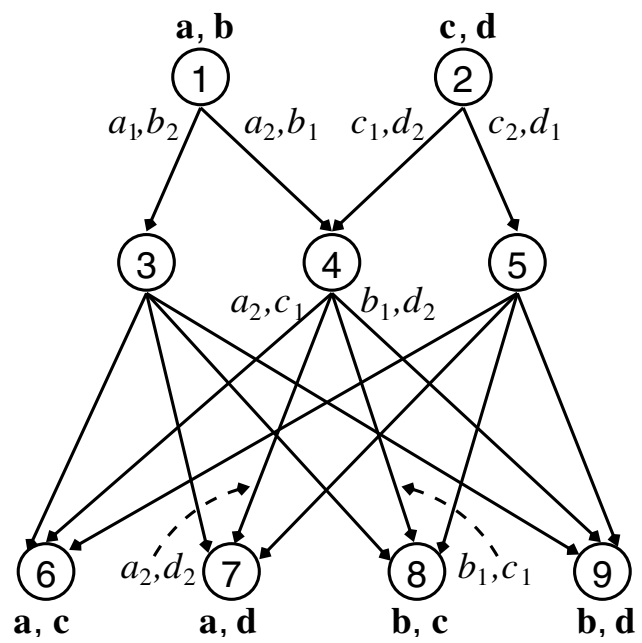

Fig. 4. The network $\mathcal{N}_{4}$ whose routing capacity is 1 .

If $\mathbf{x}$ is a message and $j$ is the unique index in a minimal $(k, n)$ fractional routing solution such that every edge carrying a component $x_{i}$ appears in $T_{j}^{\mathbf{x}}$, then we say the $\mathbf{x}$-tree $T_{j}^{\mathbf{x}}$ carries the message component $x_{i}$. Such a tree is guaranteed to exist since each message component must be routed from its source to every destination node demanding the message, and the minimality of the solution ensures that the edges carrying the message form an $\mathbf{x}$-tree.

If $\mathbf{x}$ and $\mathbf{y}$ are distinct messages and $T_{i}^{\mathbf{x}}$ and $T_{j}^{\mathbf{y}}$ are topologically the same directed subgraph of the network, then we make the convention that $T_{i}^{\mathbf{x}} \neq T_{j}^{\mathbf{y}}$. That is, such trees are determined by their topology together with their associated message.

Denote by $T_{i}$ the $i^{\text {th }}$ tree in some fixed ordering of the set

$$
\bigcup_{\mathbf{x} \in M}\left\{T_{1}^{\mathbf{x}}, \ldots, T_{s(\mathbf{x})}^{\mathbf{x}}\right\}
$$

and define the following index sets:

$$
\begin{aligned}
& A(\mathbf{x})=\left\{i: T_{i} \text { is an } \mathbf{x} \text {-tree }\right\} \\
& B(e)=\left\{i: T_{i} \text { contains edge } e\right\} .
\end{aligned}
$$

Note that the sets $A(\mathbf{x})$ and $B(e)$ are determined by the network, rather than by any particular solution to the network. 
Denote the total number of trees $T_{i}$ by $t=\sum_{\mathbf{x} \in M} s(\mathbf{x})$. We refer to the following four conditions as the network inequalities associated with a given network:

$$
\begin{array}{ll}
\sum_{i \in A(\mathbf{x})} d_{i} \geq 1 & (\forall \mathbf{x} \in M) \\
\sum_{i \in B(e)} d_{i} \leq \rho & (\forall e \in E) \\
0 \leq d_{i} \leq 1 & \\
0 \leq \rho \leq t &
\end{array}
$$

where $d_{1}, \ldots, d_{t}, \rho$ are real variables. If a solution $\left(d_{1}, \ldots, d_{t}, \rho\right)$ to the network inequalities has all rational components, then it is said to be a rational solution.

Lemma 3.1: If a non-degenerate network has a minimal fractional routing solution with achievable routing rate $r>0$, then the network inequalities have a rational solution with $\rho=1 / r$.

Lemma 3.2: If the network inequalities corresponding to a non-degenerate network have a rational solution with $\rho>0$, then there exists a fractional routing solution to the network with achievable routing rate $1 / \rho$.

We use the network inequalities to prove that the routing capacity of a network is achievable. To prove this property, the network inequalities are viewed as a set of equations in $t+1$ variables, $d_{1}, \ldots, d_{t}, \rho$, which one can attempt to solve. By formulating a linear program, it is possible to determine a fractional routing solution to the network which achieves the routing capacity.

Theorem 3.3: The routing capacity of every non-degenerate network is achievable.

Corollary 3.4: The routing capacity of every network is rational.

Corollary 3.5: There exists an algorithm for determining the routing capacity of a network.

Theorem 3.6: For each rational $r>0$ there exists a solvable network with routing capacity $r$.

\section{CODING CAPACITY}

The concept of the routing capacity of a network is now generalized to the coding capacity of a network. A $(k, n)$ fractional coding solution of a network is a coding solution that uses messages with $k$ components and edges with capacity $n$. If a network has a $(k, n)$ fractional coding solution, then the rational number $k / n$ is said to be an achievable coding rate. The coding capacity is then defined to be

$$
\gamma=\sup \{r \in \mathbb{Q}: r \text { is an achievable coding rate }\} .
$$

If a $(k, n)$ fractional coding solution uses only linear coding, then $k / n$ is an achievable linear coding rate and we define the linear coding capacity to be

$$
\lambda=\sup \{r \in \mathbb{Q}: r \text { is an achievable linear coding rate }\} .
$$

Clearly, for a given alphabet, the coding capacity of a network is always greater than or equal to the linear coding capacity. Also, if a network is solvable (i.e. with $k=n$ ), then the coding capacity is greater than or equal to 1 , since $k / n=k / k$ is an achievable coding rate. Similarly, if a network is linearly solvable, then the linear coding capacity is greater than or equal to 1 .

Example 4.1: As considered in Example 2.1, the network $\mathcal{N}_{1}$ has routing capacity $\epsilon=3 / 4$. We can show that both the coding and linear coding capacities are equal to 1 , which is strictly greater than the routing capacity.

Note that unlike fractional routing solutions, fractional coding solutions must be considered in the context of a specific alphabet. Indeed, the linear coding capacity in general depends on the alphabet [3]. However, the following theorem can be proven.

Theorem 4.2: The coding capacity of any network is independent of the alphabet used.

\section{ACKNOWLEDGMENT}

This work was supported by the Institute for Defense Analyses, the National Science Foundation, and Ericsson.

\section{REFERENCES}

[1] R. Ahlswede, N. Cai, S.-Y. R. Li, and R. W. Yeung, "Network information flow," IEEE Transactions on Information Theory, vol. 46, no. 4, pp. 1204-1216, July 2000.

[2] J. Cannons, R. Dougherty, C. Freiling, and K. Zeger, "Network routing capacity," IEEE/ACM Transactions on Networking, (submitted October $16,2004)$.

[3] R. Dougherty, C. Freiling, and K. Zeger, "Insufficiency of linear coding in network information flow," IEEE Transactions on Information Theory (revised January 6, 2005).

[4] S.-Y. R. Li, R. W. Yeung, and N. Cai, "Linear network coding," IEEE Transactions on Information Theory, vol. 49, no. 2, pp. 371-381, February 2003.

[5] M. Médard, M. Effros, T. Ho, D. Karger, "On coding for non-multicast networks," 41st Annual Allerton Conference on Communication Control and Computing, Illinois, October 2003.

[6] A. Rasala Lehman and E. Lehman, "Network information flow: Does the model need tuning?," Symposium on Discrete Algorithms (SODA), January 2005. 